\title{
4 \\ EL ‘55 EN LA UNIVERSIDAD NACIONAL DEL LITORAL. REFORMISMO Y DESPERONIZACIÓN EN EL DISCURSO DE LAS AUTORIDADES
}

Pablo Salomon 


\section{RESUMEN}

Entre 1955 y 1958, durante la intervención del Poder Ejecutivo Nacional en la Universidad Nacional del Litoral, se desplegó un proceso de desperonización que involucró un conjunto de acciones. Entre ellas, cesantías y renuncias de docentes que habían tenido vinculaciones con el régimen peronista y reincorporaciones de aquellos cuyas carreras universitarias fueron interrumpidas en 1946. Asimismo, se iniciaron reestructuraciones institucionales que buscaron borrar los símbolos alegóricos del peronismo. Este artículo, precisamente, aborda una dimensión de este proceso al analizar el discurso de las autoridades interventoras. En la interpretación que las autoridades realizaron de esa coyuntura crítica, apelaron al reformismo para la consecución de la desperonización en la casa de altos estudios.

\section{ABSTRACT}

Between 1955 and 1958, during the intervention of the national government to the Universidad Nacional del Litoral, a sistematic process of elimination of contents, ideas and symbols from the Peronist period (called in Spanish «desperonización») involved a set of actions. Professors who had links with the Peronist regime were removed or resigned their positions. Those whose university careers were interrupted in 1946 were reincorporated. In addition, institutional restructuring was initiated to erase allegorical symbols of Peronism. This article, precisely, addresses a dimension of this process by analyzing the discourse of the intervening authorities. The authorities' interpretation about this critical juncture appealed to reformism in order to achieve the elimination of Peronism from the university.

\section{PALABRAS CLAVE}

$>$ Reformismo

$>$ intervención

$>$ desperonización

$>$ Universidad Nacional del Litoral

$>$ discurso

\section{KEYWORDS}

$>$ Reformism

$>$ intervention

$>$ «desperonización»

$>$ Universidad Nacional del Litoral

$>$ discourse 


\section{INTRODUCCIÓN}

El 2 de abril de 1956, al volver a la Universidad Nacional del Litoral (UNL) luego de su exilio en Uruguay, el profesor Rudesindo Martínez, pronunció unas palabras en el acto de apertura del año académico. ${ }^{1}$ Desde el recinto del Paraninfo de la Universidad, se refirió al momento político que se estaba viviendo y sostuvo que:

Después de doce años de forzada ausencia, ocupo con honda emoción esta tribuna que ha vuelto a ser, por imperio de los acontecimientos, la cátedra libre, de una universidad reformista de un pueblo libre. No podríamos, en otras condiciones, reanudar nuestra labor docente quienes nos incorporamos a esta casa de estudios bajo el signo de la reforma universitaria iniciada allá, por los inolvidables días de 1918, en Córdoba, la docta y tradicional, donde se inició también hace unos meses el movimiento que, tras gloriosas jornadas, ha liberado al país de una dictadura más sombría y regresiva que la de Rosas. ${ }^{2}$

Este fragmento es ilustrativo de la manera en que la apelación a la tradición reformista se articuló con las primeras acciones de la Revolución Libertadora en el ámbito universitario. Por ello, con el propósito de esclarecer la relación entre reformismo y desperonización, en este artículo focalizamos el discurso de las autoridades que asumieron la conducción de la UNL a partir del de $1955 .^{3}$

\footnotetext{
${ }^{1}$ Bajo el título «Fueron agasajados en esta capital dos exiliados en el Uruguay» se describía la llegada de Rudesindo Martínez a la ciudad de Santa Fe luego de su residencia en Montevideo, señalando que habían sido recibidos por una comitiva del partido radical. Diario El Litoral, Santa Fe, 04/10/1955.

2 Revista Universidad, (32), junio de 1956, 33.

${ }^{3}$ En otro trabajo hemos abordado el proceso de desperonización en la UNL cuantificando las cesantías, renuncias y reincorporaciones que se sucedieron en los años de la intervención. Asimismo, hemos atendiendo a las reestructuraciones institucionales que buscaban borrar los símbolos alegóricos del peronismo (Salomon, 2014).
} 


\section{GOLPE DE ESTADO \\ E INTERVENCIÓN A LAS UNIVERSIDADES}

Como es sabido, el golpe de Estado producido en 1955 fue apoyado por un amplio frente de fuerzas políticas y sociales que incluía al conjunto del arco político partidario opositor al peronismo, las asociaciones patronales, la iglesia católica y las federaciones de estudiantes universitarios.

Qué hacer con el peronismo fue una las cuestiones claves a resolver por el gobierno militar. En este sentido, el primer presidente de facto, el general Lonardi, en su breve gestión, abordó la «cuestión peronista» desde una posición conciliadora expresada en la consigna que proclamaba que, en la Argentina, no había «ni vencedores ni vencidos». Luego de este comienzo vacilante, la sucesión presidencial que desplazó a Lonardi y llevó a Aramburu al poder, consolidó la postura de los sectores más radicalizados del antiperonismo. Esta posición se tradujo en la proscripción al peronismo y la intervenían a la central obrera, medidas que se alejaban de cualquier neutralidad respecto del pasado inmediato (Tcach, 2003; Spinelli, 2003). En ese contexto, el gobierno decidió intervenir las universidades nacionales para iniciar un proceso de reestructuración, que tendría como uno de sus puntos nodales la desperonización de las casas de altos estudios.

Entre las primeras medidas desplegadas por el gobierno militar cabe destacar la derogación de las leyes universitarias sancionadas durante el gobierno peronista (31031/47 y 14297/54). En general, ambas leyes establecían la designación directa de los rectores por parte del presidente de la Nación, la elección de los decanos exclusivamente por el claustro docente (a partir de una terna elaborada por el rector), la representación estudiantil en los consejos académicos en la figura de un alumno avanzado (seleccionado por las autoridades universitarias conforme a criterios meritocráticos) y la elección de los docentes titulares por parte del Poder Ejecutivo Nacional, sobre la base de una terna proporcionada por las universidades previo concurso de antecedentes. Además se prohibía la actuación política de los miembros de la comunidad universitaria (Pronko, 2000; Soprano, 2009; Mangone y Warley, 1984).

La normativa que reemplazó a ambas leyes fue el decreto-ley 6304/55 que contenía las bases para la reorganización de todas las casas de altos estudios del país. En dicho decreto se establecieron los principales lineamientos a seguir, 
entre ellos, la recuperación de la autonomía universitaria, la democratización de la conducción a partir del cogobierno de docentes, estudiantes y graduados, la autarquía y el manejo del patrimonio, la sanción de sus propios estatutos, las reformas de los planes de estudio y la elección de los docentes sin injerencia estatal (Buchbinder, 2005).

Al mismo tiempo, como parte del proceso de reestructuración universitaria se decretó la «puesta en comisión» del personal docente de las universidades y, como forma de "reparación moral» ${ }^{4}$ (Mignone, 1998), se estableció la reincorporación de los profesores que hubiesen sido expulsados de sus cátedras durante el gobierno peronista. Asimismo, se establecieron mecanismos para obstaculizar la permanencia o ingreso de profesores que tuvieran alguna vinculación con el peronismo. Así, quienes quisieran ingresar a la universidad deberían tener «Conducta moral inobjetable (...) No serán admitidos quienes hayan realizado actos positivos y ostensibles que prueben objetivamente la promoción de doctrinas totalitarias [y quienes hubieran] realizado actos positivos y ostensibles de solidaridad con la dictadura». ${ }^{5}$

Mientras se producía el derrocamiento de Perón, en las ciudades de Santa Fe, Rosario y Paraná, diversos actores universitarios, fundamentalmente los estudiantes, a través de movilizaciones, asambleas y tomas de los edificios de distintas facultades manifestaron su oposición a la universidad gobernada por el peronismo. ${ }^{6}$ En ese marco, la UNL fue intervenida en octubre de 1955. El Dr. José María Manuel Fernández fue designado en el cargo de rector interventor y fue acompañado por el Dr. Domingo Buonocore, quien ejerció los cargos de vicerrector y de decano interventor de la Facultad de Ciencias Jurídicas y Sociales. Ambas autoridades interventoras habían desarrollado trayectorias académicas e institucionales semejantes.

José Fernández inició su vínculo con la UNL en 1935 cuando comenzó a dictar la cátedra Dermatosifilográfica en la Facultad de Ciencias Médicas, accediendo a la titularidad en 1940. Paralelamente comenzó sus investigaciones

\footnotetext{
${ }^{4}$ Decreto $2538,11 / 10 / 1955$.

${ }^{5}$ Decreto 6304/55.

${ }^{6}$ Véanse los periódicos de la ciudad de Santa Fe, El Litoral y El Orden, y los de Rosario, La Capital, Democracia, y Acción (desde el 16/09/1955 hasta el 3/10/1955).
} 
sobre la lepra. Al producirse el golpe de Estado de 1943 y la intervención en la UNL a cargo de Jordán Bruno Genta -exponente del nacionalismo católico-, Fernández renunció a su cargo. No obstante, en marzo de 1945 fue reincorporado y designado como delegado suplente de la facultad ante el Consejo Superior de la UNL. ${ }^{7}$

Su legajo personal registra que el Consejo Directivo de la Facultad de Ciencias Médicas decidió «agregar a sus antecedentes, la reseña de su destacada actuación científica desarrollada durante el tiempo que permaneció alejado de la facultad». ${ }^{8}$ En 1945 fue designado delegado de la facultad para dictar conferencias en el Círculo de Medicina de San Luis y Mendoza. A principios de 1946 se le otorgó una licencia para asistir a Brasil a una reunión científica de la Sociedad Paulista de Leprosos. Pero en noviembre de 1946, en el marco de una nueva intervención a la UNL decretada por el presidente Perón, fue nuevamente separado de su cargo ${ }^{9}$ (Salomon, 2014).

Por su parte, el vicerrector, Domingo Buonocore, fue otro universitario relevante en el contexto político-institucional abierto por la Revolución Libertadora. Su trayectoria muestra una fuerte ligazón con la Facultad de Ciencias

${ }^{7}$ Como es sabido, entre 1943 y 1946 la Argentina estuvo gobernada por un régimen militar. En sus inicios predominaron las políticas de corte autoritario y con una tendencia ideológica que abrevaba en el nacionalismo católico. En el marco del conflicto internacional de la Segunda Guerra Mundial, este régimen planteó una política de neutralidad hacia el conflicto que era interpretada como una cierta admiración hacia las potencias del Eje. Pero a comienzos de 1945, cuando la derrota de Alemania y sus aliados era inexorable, el régimen militar argentino decidió ajustar sus políticas a los nuevos tiempos. En este sentido, en el plano interno reinstaló las libertades públicas, los partidos políticos volvieron a la legalidad y de las universidades se retiraron los gobiernos interventores que habían buscado tener un rígido control en cuestiones políticas e ideológicas.

${ }^{8}$ Acta No 203/1945, Ficha Docente.

${ }^{9}$ Luego de alejarse de la UNL, Fernández fundó una clínica para el tratamiento de enfermedades de la piel en Rosario y continuó con sus investigaciones sobre la lepra. Por esos años, junto a colegas de Brasil, sentó las bases para una nueva clasificación de la lepra que fue reconocida en 1948 en el V congreso Internacional de Lepra realizado en Cuba y sancionada en el congreso de Madrid realizado en 1953. Además, en 1947 el gobierno de Perú lo contrató como asesor para realizar una campaña antileprosa en ese país. También, en 1950 la Organización Mundial de la Salud organizó un comité de expertos en lepra nombrándolo a Fernández entre sus ocho miembros; no obstante, las autoridades argentinas no dieron el visto bueno para que integrara dicho comité (Molinari, 2008). 
Jurídicas y Sociales, que llegó a presidir como decano a mediados de los años cincuenta. La relación se había iniciado con el ejercicio de la docencia desde la década del '30. ${ }^{10} \mathrm{~A}$ la par de sus actividades docentes, realizó otras funciones: en 1931 fue designado redactor de la Revista de Ciencias Jurídicas y Sociales; en 1935, director de la Biblioteca y entre 1939 y 1946 desempeñó tareas como secretario de la revista Universidad.

Al mismo tiempo, cabe destacar que Buonocore formó parte del gobierno de la facultad ya que fue electo consejero directivo por el estamento de los profesores en 1932, 1936 y 1937. Al igual que el rector interventor, tuvo una relación conflictiva con la intervención de 1943 ya que fue suspendido en sus cargos por enfrentarse con las autoridades de ese momento. ${ }^{11}$ La suspensión duró poco tiempo, hasta fines del año 1943. Pero, en octubre de 1946, en el contexto propiciado por una nueva intervención, fue apartado nuevamente de sus cargos en la facultad (Salomon, 2014, 2016).

En 1955, coherente con su trayectoria, el rector interventor José Fernández hizo explícita su posición sobre la situación vivida por las universidades argentinas durante los años de gobierno peronista. Al respecto planteó:

(...) deseo en primer lugar rendir mi emocionado homenaje a los profesores y personal docente que sufrieron persecución sin doblegarse, manteniendo inmaculado su decoro y dignidad frente a la prepotencia, lo mismo a esa juventud heroica (...) a ese magnífico estudiantado de nuestras Universidades, que se mantuvo incorruptible frente a la coacción y al soborno, dándonos un ejemplo conmovedor de la firmeza de sus ideales. ${ }^{12}$

Sus dichos anticipaban el rumbo que adoptaría la intervención, sobre todo en relación con las medidas que buscaban desperonizar la universidad.

\footnotetext{
10 Ejerció la docencia en dos áreas del Derecho: en 1930 fue designado profesor suplente en Derecho Administrativo, y en 1934 se desempeñó interinamente en la cátedra de Derecho Agrario Rural y de Minas. Para 1940 su carrera docente se había consolidado en ambas cátedras, como adjunto y titular respectivamente. Legajo Docente y Abad de Santillán (1963).

${ }^{11}$ Resolución No 59, 22/9/1943.

12 Revista Universidad, (31), diciembre de 1955, 17.
} 


\section{REFORMISMO Y DESPERONIZACIÓN EN LA VOZ DE LAS NUEVAS AUTORIDADES}

En distintas conferencias e intervenciones públicas, las nuevas autoridades universitarias se pronunciaron sobre el contexto político que se abría con el derrocamiento del peronismo y los caminos que la UNL debía transitar a partir de la intervención. Estos pronunciamientos se desarrollaron al calor de uno de los debates claves de esos años, que se puede condensar en una sola pregunta: ¿Qué es el peronismo? Conocer y caracterizar este fenómeno político fue una de las maneras que tuvieron los universitarios para diagramar las posibles alternativas a seguir por parte de la institución del litoral.

En la revista Universidad, órgano político-cultural de la UNL, ${ }^{13}$ se publicaron las conferencias de quienes asumieron cargos directivos en la coyuntura abierta por el golpe de 1955. Es por esto que, desde sus páginas, podemos aproximarnos a los avatares del proceso político abierto con la Revolución Libertadora.

La publicación del número $31^{\circ}$ de la revista coincidió con ese momento álgido. Con el discurso "La recuperación de la Universidad» ${ }^{14}$ del ministro de Educación, Alitio Dell'Oro Maini, quedaron expresados los lineamientos de la nueva política universitaria. Por su parte, el rector interventor de la UNL explicitaba su «Programa de acción», destacando que «venimos simplemente a preparar el terreno para esa gran tarea (la reestructuración) que será obra exclusiva de cada Universidad». ${ }^{15}$

En 1956 se organizó un acto a propósito de la inauguración del ciclo lectivo. En sus discursos, publicados en el número 32 de la revista Universidad, las autoridades de la UNL expusieron la situación por la que atravesaban las universidades. El acto y las intervenciones fueron una tribuna política para impugnar las acciones de quienes habían gobernado la universidad durante el peronismo. Hablaron el rector interventor, José María Manuel Fernández; el

\footnotetext{
${ }^{13}$ En esta revista se publicaban periódicamente artículos de las diversas ramas del conocimiento a la vez que se divulgaban las posturas tomadas por la universidad y sus autoridades con respecto a diversas cuestiones que afectaban a la sociedad argentina.

${ }^{14}$ Revista Universidad, (31), diciembre de 1955, 7.

15 Ídem, 18.
} 
vicerrector y decano-interventor de la Facultad de Ciencias jurídicas y Sociales, Domingo Buonocore; el recientemente reincorporado profesor de dicha facultad, Rudesindo Martínez, y el decano y profesor de la Facultad de Derecho de Montevideo, Eduardo J. Couture.

Las «Palabras preliminares» del número 32 de la revista son más que elocuentes para sentar posición frente a la experiencia peronista en la universidad. Las nuevas autoridades interpretaban que el gobierno peronista había sido una dictadura y trasladaban esa interpretación a su gestión universitaria:

Después de una interrupción de diez años — pausa larga y dolorosa para la cultura argentina - retomamos el gobierno de la revista UNIVERSIDAD. En el lapso de tiempo que corre entre el 2 de mayo de 1946 - fecha en que todas las universidades del país fueron avasalladas simultáneamente por un decreto inaudito- hasta el 21 de septiembre de 1955, día que señala el advenimiento de la Revolución Libertadora, la universidad argentina, reiterada y torpemente agraviada en sus fueros y tradiciones, sufrió el vejamen más grande que registró la historia. ${ }^{16}$

El discurso pronunciado por Domingo Buonocore, quien se proponía reflexionar sobre «La enseñanza del derecho en las universidades de Argentina y Uruguay», destacaba la importancia que tuvo la universidad uruguaya para los docentes que habían sido expulsados de sus cátedras bajo el régimen peronista: «Nunca olvidaremos que, allende el Plata, los profesores argentinos sin cátedras encontraron, en la hora amarga de la dictadura, un refugio para sus desvelos e inquietudes». ${ }^{17}$

Los universitarios del litoral apelaban al pasado para significar la experiencia peronista y la equiparaban con la «dictadura rosista». Por consiguiente, ese recorte selectivo del pasado que era, a su vez, una lectura política del presente, permitía valorar positivamente tanto el acontecimiento de la batalla de Caseros, que había provocado el derrocamiento del rosismo, como la Revolución Libertadora que había puesto fin a la experiencia peronista. Así lo expresaba Buonocore:

\footnotetext{
${ }^{16}$ Revista Universidad, (32), junio de 1956, 7.

17 Ídem, 13.
} 
la tierra uruguaya es tierra de bendición para los argentinos. En ella velaron sus armas los hombres que habrían de sellar la libertad en la mañana luminosa de Caseros (...). Nunca olvidaremos los hijos de la tierra de San Martín que cuando el horizonte de la patria se oscureció, fue el pensamiento libre y el espíritu insobornable de los uruguayos, la única luz y la única fuerza desde el exterior que se pusieron al servicio de la lucha por la recuperación de la dignidad perdida. Así como en la nueva Troya se consolidó durante los nueve años luctuosos de su sitio, el núcleo de resistencia que habría de derribar a Rosas, así también, a la vuelta de más de un siglo (...) Montevideo se convertiría, una vez más, en el baluarte de la libertad y en asilo de argentinos perseguidos en su patria. ${ }^{18}$

Lo dicho por Buonocore se corresponde con la decisión por parte de las autoridades de participar en los festejos por la «gesta de Caseros», ya que «la Universidad no puede permanecer ajena a esta recordación, en momentos de tanta trascendencia para la Nación». El 3 de febrero de 1956 se recordaría la batalla que había terminado con el régimen de Rosas y, según se expuso en la resolución rectoral, esta había permitido «el advenimiento de otra era de afianzamiento democrático, organización nacional, paz y progreso como la idearon nuestros próceres de Mayo». ${ }^{19}$ Esta forma de recordar funcionaba como una metáfora de la interpretación del derrocamiento del peronismo. Como señala Beatriz Sarlo, en la coyuntura de 1955, los significados atribuidos al siglo XIX iban «ligados a la convicción de que se asistía a un momento que era a la vez de clausura y de inauguración» (Sarlo, 2001:19).

Como ha sido señalado por numerosas investigaciones, el gobierno de Perón - con la instalación de los Cursos de Formación Política, el Instituto del Justicialismo, la imposición de los nombres de Perón y Eva Perón a distintos espacios institucionales - buscó partidizar la Universidad minimizando su autonomía. Cuando sobrevinieron el golpe y la intervención, la nueva gestión universitaria desarmó esos dispositivos institucionales e impugnó la modalidad de partidización que la universidad había adoptado durante el gobierno peronista. Por ello, Buonocore consideraba que:

\footnotetext{
18 Ídem, 10-11.

${ }^{19}$ Resolución Rectoral, 1/02/1956.
} 
la educación no puede ni debe estar constreñida unilateralmente a la especialidad profesional (...) sino que debe mirar, por sobre todo, a la formación integral del hombre libre para actuar en una República libre. Mal puede formarse el hombre libre en el seno de una universidad militante o sectaria como fue la universidad argentina durante la época aciaga de la dictadura. Esa universidad se traicionó a sí misma para asumir una orientación partidista incompatible con el principio de objetividad del espíritu científico. ${ }^{20}$

Pero esto no significaba despolitizar la universidad para aislarla del conjunto de la sociedad, sino que se buscaba superar la impronta del peronismo para replantear y redefinir la política universitaria. El grupo que dirigió la Universidad a partir de la intervención consideraba que esta institución debía vincularse con el desarrollo del país, rechazando la idea de una universidad en una posición expectante o de neutralidad frente a los problemas sociales. Es por esto que los actores irían delimitando y definiendo el horizonte que debía alcanzar esta nueva relación, que se expresaba en los siguientes enunciados del discurso del vicerrector:

ni universidad beligerante ni universidad expectante. Los dos extremos conspiran igualmente contra la esencia de la institución y sus fines específicos (...) Si no es admisible el aislamiento estéril, porque forma profesionales extraños a la sociedad y a su tiempo, ni la militancia activa porque aniquila el espíritu científico, la universidad tiene que asumir una actitud de intervención frente a los hechos y fenómenos de la realidad. Debe captarlos, examinarlos, determinar sus causas, formular las teorías explicativas (...) pero con método y ánimo rigurosamente imparcial. De esta manera, la universidad se convierte en actora y rectora, pero entendiendo que le está vedado tomar partido y mezclarse en las luchas e interés de la calle. ${ }^{21}$

Con este posicionamiento, quienes se hicieron cargo de la UNL a partir del golpe de 1955 buscaban restablecer la tradición del reformismo universitario,

\footnotetext{
${ }^{20}$ Revista Universidad, (32), junio de 1956, 15.

21 Ídem, 16.
} 
tradición que, desde su perspectiva, el peronismo había desvirtuado. Lo que se buscaba dejar en el pasado era la universidad que había perdido su autonomía, que se había organizado como una dependencia administrativa del régimen de gobierno, que había intentado hacerla parte de su dispositivo simbólico.

Rudesindo Martínez fue otro de los profesores que, desde la tribuna del paraninfo de la UNL, se manifestó sobre el contexto político abierto con la intervención en 1955. Graduado como abogado en la Universidad de Buenos Aires, desde 1921 se vinculó con la UNL cuando fue nombrado profesor en la cátedra Historia Diplomática (luego denominada Derecho Internacional Público) y en 1936, en Filosofía del Derecho. Su relación con el gobierno de la Facultad de Ciencias Jurídicas y Sociales se afianzó desde en 1923, cuando fue electo consejero directivo, reelecto en 1930, y en 1934 fue designado representante de la facultad ante el Consejo Superior.

Con la intervención de 1943 Martínez fue separado de sus cátedras hasta septiembre de 1945, cuando el gobierno militar flexibilizó su política inicialmente autoritaria reinstalando las libertades públicas y «retirándose» del control sobre las universidades. Pero en 1946 fue separado nuevamente de sus funciones por una década. Con la intervención de 1955 fue reincorporado a sus cátedras universitarias y en 1958 resultó electo consejero directivo de la Facultad de Ciencias Jurídicas y Sociales.

Su discurso de 1955 se destaca por componer un relato de la historia de la UNL, poniendo de relieve ciertos hitos institucionales. En primer lugar, subrayaba que la nacionalización de la Universidad del Litoral en 1919 se produjo bajo el influjo de las ideas dominantes de la Reforma Universitaria de 1918. Resaltaba la discusión y sanción, por parte de la dirigencia universitaria, del Estatuto propio en 1936. Cuestionaba duramente la ideología que había pretendido imponerse con la intervención decretada en 1943 por entender que destruía las bases estructurantes de la universidad. Esgrimiendo la tradición reformista del estatuto de 1936, ponía en cuestión las ideas de Jordán Bruno Genta al asumir el cargo de rector interventor de la UNL en 1943. Ciertos fragmentos del discurso son reveladores del contrapunto con las ideas de Genta: 
Cuando en 1943 (...) se dijo, desde esta misma tribuna, que el problema del momento era una simple cuestión entre antiguos y modernos (...) se dirigió un violento ataque contra la cultura superior y el progreso individual y social, máxima finalidad de esta institución, de acuerdo a lo prescripto en los estatutos que en ejercicio de su autonomía se había dado en 1936. (...) después de acusarla de ser una universidad sin metafísica y sin nacionalidad, intentó hacer una crítica demoledora del sentido y fines de esta institución, enunciados clara y precisamente en el artículo $1^{\circ}$ de sus estatutos. Tras de afirmar que las ciencias positivas carecen de patria y de definición moral, se dijo desde aquí que la expresión «cultura superior» no pasaba de ser una vaguedad retórica (...). Con esa condenación del progreso y de la democracia, con esa valoración despectiva de las ciencias (...) se inició un violento ataque declamatorio contra las universidades modernas. ${ }^{22}$

En la reconstrucción histórica realizada por Martínez la intervención de 1943 constituía una bisagra en la vida institucional. El acontecimiento era percibido como la clausura de una primera etapa y el comienzo de otra que tendría continuidad en los años de gobierno peronista:

Lo que perseguía, en verdad, era destruir la universidad autónoma y la libertad de cátedra, para estructurar una universidad servil, prosternada ante el dictador e incondicionalmente utilizable para la difusión de una doctrina totalitaria elaborada con residuos del nazifacismo. ${ }^{23}$

En la apelación al reformismo que realizaban las autoridades de la UNL se puede identificar esa «naturaleza mixta, al mismo tiempo cultural y política, proyecto para la Universidad y voluntad de cambio para el país. (...) que siempre tuvo un pie en la Universidad y un pie fuera de ella, en la sociedad» (Sigal, 2002:60). Esa manera de entender la intervención social de la Universidad mantenía a resguardo la propia identidad de los universitarios, lo que se expresaba en las posiciones asumidas en el marco de su consolidación en la cúspide del poder universitario.

\footnotetext{
22 Revista Universidad, (32), junio de 1956, 36-39.

23 ídem, 39.
} 


\section{A MODO DE CIERRE}

En el contexto de la Revolución Libertadora, el discurso de las nuevas autoridades universitarias enfatizó, en términos generales, el cuestionamiento al gobierno peronista y, en particular, a la manera de gestionar las universidades. Desde el posicionamiento en la tradición reformista y en los principios consagrados en la Córdoba de 1918, se impugnaron los efectos de lo que se interpretaba como «una dictadura más sombría y regresiva que la de Rosas», al decir de Rudesindo Martínez. De este modo, la reestructuración universitaria, cuyos pilares eran la recuperación de la autonomía y la democracia interna, representadas en el cogobierno de docentes, estudiantes y graduados, conllevaba la exclusión de todos los contenidos, ideas y símbolos vinculados con el peronismo.

\section{REFERENCIAS BIBLIOGRÁFICAS}

Buchbinder, P. (2005). Historia de las universidades argentinas. Buenos Aires: Sudamericana.

Mangone, C. y Warley, J. (1984). Universidad y peronismo (1946-1955). Buenos Aires: CEAL.

Mignone, E. (1998). Política y Universidad. El estado legislador. Buenos Aires: Lugar Editorial.

Molinari, I. (2008). El Dr. José María Manuel Fernández. Medicina y ciencia al servicio de la erradicación del Mal de Hansen. En Álvarez, A. y Carbonetti, A. Saberes y prácticas médicas en la Argentina. Un recorrido por historias de vida (pp. 225-251). Mar del Plata: Editorial de la Universidad Nacional de Mar del Plata.

Pronko, M. (2000). El peronismo en la Universidad. Buenos Aires: Libros del Rojas. Salomon, P. (2014). Intervención, desperonización y elencos de gobierno. La Universidad Nacional del Litoral entre 1955-1958. Papeles del Centro de Investigaciones, 4(15), 13-34. Santa Fe: Facultad de Ciencias Jurídicas y Sociales, UNL. 
(2016). Trayectorias y elencos de gobierno. La Facultad de Ciencias Jurídicas y Sociales en los años sesenta. En: Sozzo, G. (Dir.). Hacer Derecho: reconstrucciones acerca de la relación derecho/ciencias sociales en la Facultad de Ciencias Jurídicas y Sociales de la Universidad Nacional del Litoral (pp. 169-184). Santa Fe: Ediciones UNL. Sarlo, B. (2007). La Batalla de la Ideas (1943-1973). Buenos Aires: Emecé.

Sigal, S. (2002). Intelectuales y poder en la Argentina. La década del sesenta. Buenos Aires: Siglo XXI Editores.

Soprano, G. (2009). Política, instituciones y trayectorias académicas en la universidad argentina. Antropólogos y antropología en la UNLP entre las décadas de 1930 y 1960. En Marquina, M., Mazzola, C. y Soprano, G. (Comps.). Políticas, instituciones y protagonistas de la universidad argentina (pp. 111-152). Buenos Aires: Prometeo. Spinelli, M.E. (2003). Ideas fuerza en el debate político durante los años de la «Libertadora», 1955-1958. Estudios Sociales. Revista universitaria semestral, XIII(24), primer semestre, 61-88. UNL, Santa Fe.

Tcach, C. (2003). Golpes, proscripciones y partidos políticos. En James, D. (Dir.). Violencia, proscripción y autoritarismo (1955-1976). Nueva Historia Argentina. Tomo IX (pp. 17-62). Buenos Aires: Sudamericana.

Torre, J.C. (2003). Introducción a los años peronistas. En Los años peronistas. 19431955. Nueva Historia Argentina. Tomo VIII (pp. 11-77). Buenos Aires: Sudamericana. 